\title{
Peduncular Hallucinosis: An Unusual Case of Visual Hallucination in a Delirious Patient
}

\author{
Krishnendu Ghosha, ${ }^{\mathrm{a}}$, Guitelle St. Victor ${ }^{\mathrm{a}}$
}

\begin{abstract}
Visual hallucination is very common in case of delirium and other neuropsychiatric disorders. It is one of the most common clinical symptoms for which psychiatric consultation is requested on medical floors. When hallucination dominates the clinical presentation, a patient may be misdiagnosed as suffering from a psychiatric disorder. This case is a wonderful example of Peduncular Hallucinosis, an underlying organic pathology. A possible pontine tumor was discovered in a patient admitted to the medical floor with altered mental status in the absence of any focal neurological signs.
\end{abstract}

Keywords: Visual hallucination; Peduncular hallucination; Magnetic resonance imaging

\section{Introduction}

When a patient presents with visual hallucination in the ER, we first consider the most common conditions like delirium, dementia or psychosis. Visual hallucinations can originate from a variety of psychiatric, medical, neurologic, ophthalmologic and metabolic disorders. A study done in a geriatric psychiatric clinic found that $10 \%$ of the clinic patients experienced visual hallucinations [1]. A classic study of delirium, by Wolff et al [2] noted that visual hallucinations were present in $67.9 \%$ of delirious patients. Another study by Webster [3] showed that $27 \%$ of patients with delirium experienced visual hallucinations. The importance of correctly identify-

Manuscript accepted for publication September 24, 2012

${ }^{\mathrm{a}}$ Von Tauber Institute of Global Psychiatry, Department of Psychiatry and Behavioral Science, Nassau University Medical Center, NY, USA

${ }^{\mathrm{b} C o r r e s p o n d i n g ~ a u t h o r: ~ K r i s h n e n d u ~ G h o s h, ~} 200$ Carman Avenue, Apt

30 A, East Meadow, NY, 11554, USA. Email: Kghoshmd@yahoo.com

doi: http://dx.doi.org/10.4021/jmc868w ing the cause of this extremely distressful symptom cannot be overemphasized. In the case mentioned below, visual hallucination was found to be caused by a rare condition called peduncular hallucinosis, in a patient who presented with altered mental status.

\section{Case Report}

We report here the case of a 77-year-old African American female with past medical history of Hypertension but no prior psychiatric history. She was admitted to the medical floor with altered mental status. She was reported to have been found in a state of confusion by her family. The patient had no history of head trauma, loss of consciousness, seizure, and fever or chest pain. Her past ophthalmological history was remarkable for cataract and glaucoma. The patient reported visual hallucinations for five to six days. She had been seeing cats, dogs and small children that she knew were not real. She reported often seeing two of an item: a hallucinatory dog playing alongside of her real dog, and a child who did not really play with her great granddaughter. Sometimes she would see a man sitting on a chair next to her but he would not talk when she addressed him. She denied any auditory or other types of hallucinations. According to the patient, she had no history of hallucination prior to these experiences. She denied any symptoms of mania, psychosis or any other anxiety disorder. She felt concern about her symptoms but denied neural vegetative symptoms of depression per se. The patient had some evidence of slight cognitive decline, memory impairment, visuopatial impairment, and verbal fluency impairment. However, this did not affect her activities of daily living. The patient said that she realized driving was not safe, and hence she stopped driving. The patient's husband died three years ago of cardiac arrest. The patient had 5 children, the oldest two of which were deceased following drug overdoses in 1986 and 1989. After her daughters passed away, the patient took in their nine children and raised them as her own. The patient reported abusing alcohol from age 13 through 30 but indicated that she had been sober since age 30 . She denied use of any other recreational drugs. Patient worked as an administrative assistant 


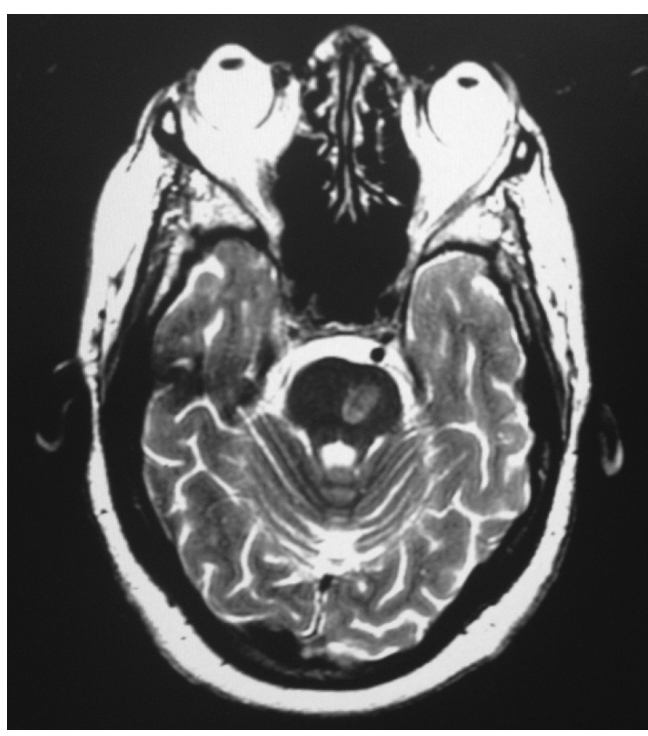

Figure 1. MRI of the brain showing scattered foci bright signal intensity on the T2 and FLAIR images in the periventricular and deep white matter, there was bright signal intensity within the left portion of the pons.

until she retired. Patient denied having a family history of any psychiatric disorder.

At the time of the interview the patient was comfortable, well groomed and appeared to her stated age. She was alert and oriented to time, place and person. She was cooperative with good eye contact. Speech was spontaneous and well-articulated. She described her mood as slightly depressed. Her affect was stable and mood congruent. No delusions were elicited. She denied any suicidal or homicidal ideation, intent or plan. Her insight was fair and judgment was appropriate. Her attention and concentration were moderately impaired as well as her short term memory. The C-L team recommended MRI of the brain, and Opthalmology and neurology consultation to rule out Charles-Bonnet syndrome.

Her general physical examination as per the primary medical team and her Neurological examination as per the Neurology team were unremarkable. Ophthalmological examination showed a Visual Acuity of 20/150 in the Right eye and 20/100 in her left eye with bilateral open angle glaucoma and cataract. Patient did not have any other ocular pathology and the ophthalmology team recommended management of cataract and glaucoma on an outpatient clinic basis. The patient's CT scan showed mild involutional changes along with ischemic-gliotic changes, without any acute intracranial pathology. Her blood tests were followed: Complete blood count showed WBC $10.7 \mathrm{~K} / \mathrm{mm}^{3}$, Hemoglobin 14.8

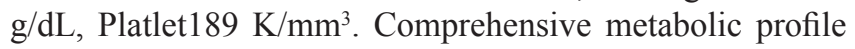
showed Sodium $141 \mathrm{mmol} / \mathrm{L}$, Potassium $4.3 \mathrm{mmol} / \mathrm{L}$, Chloride $105 \mathrm{mmol} / \mathrm{L}$, Bicarbonate $25 \mathrm{mmol} / \mathrm{L}$ with anion Gap of 13, Magnesium $2.4 \mathrm{mg} / \mathrm{dL}$, phosphorus $3.6 \mathrm{mg} / \mathrm{dL}$, Glucose
$98 \mathrm{mg} / \mathrm{dL}$, BUN $11 \mathrm{mg} / \mathrm{dL}$, and Creatinine $0.9 \mathrm{mg} / \mathrm{dL}$. LFT's showed ALT 17 U/L, AST 20 U/L, Alk Phos 92 U/L, and total bilirubin $0.3 \mathrm{mg} / \mathrm{dL}$. Coagulation Profile showed PT 12.9 secs INR 1.0, and PTT 30.2 secs.

MRI of the brain stated mild involutional changes. There were scattered foci bright signal intensity on the T2 and flair images in the periventricular and deep white matter, which could be because of ischemic-gliotic changes. No mass lesion was seen. Specifically there was a bright signal intensity within the left portion of the pons. The craniovertebral junction was unremarkable (Fig. 1).

MRA of the brain was normal. Neurology consultation addressed this as peduncular hallucinosis, with possibility of Glioblastoma multiforme in the pons. Neurology team advised MRI with contrast study to rule out any malignancy. Neurology also advised to follow-up in the neurology clinic but unfortunately patient did not show up in the clinic and lost contact.

\section{Discussion}

Visual hallucinations are caused by transient increased activity in the visual cortex. In case of visual hallucinations, the most important question arises to localize the lesion in the brain. There are two main types of visual hallucinations, formed and unformed. Unformed hallucinations consist of blotches of color, geometric shapes, flashing lights, or some other ill-defined images. Formed hallucinations, on the other hand, consist of well-defined objects, people, animals, or anything else. It is easy to figure out what type is being experienced by a patient, simply from their description. An occipital lobe focus produces lights or colors, whereas temporal lobe focus results in complex formed images [4]. The causes of formed VH include: 1) ocular pathology like macular degeneration, cataract; 2) peduncular origin, or 3) cholinergic in nature as seen in Parkinsonism. Our patient had distinctive a pontine lesion along with visual hallucination, which is the hallmark of peduncular hallucinosis.

L'hermitte first described the clinical syndrome of peduncular hallucinations, but Von Bogaert coined the term "peduncular hallucinations". Peduncular hallucination has been reported because of vascular and infective lesions of the thalamus, the pars reticulata of substantia nigra, midbrain, pons and basal diencephalon. It could be due to compression of midbrain. The etiology of the hallucinations in peduncular hallucinosis is still unknown, but causes can be vascular stroke, encephalitis, neoplastic changes, intoxication, vertebral angiography and transient brain stem compression [5-6]. Feinberg [7] and Mackee [8] explained more anatomically that occlusion of the paramedian branches of the rostral basilar artery causes infarcts in the medial diencephalon, especially pars reticulata of the substantia nigra, and brain stem tegmentum.. These regions are also con- 
nected to the pedunculopontine nuclei by efferent pathways [9]. Lesions in these areas produce disordered REM sleep, which is linked to dreams. Thus peduncular hallucinations may be similar to dream like activity that is normally suppressed during wakefulness in normal individual. Disruption of ascending impulses from brain stem reticular activating system is a postulated cause [10] of dream like activity in $\mathrm{PH}$. Whereas Von Bogert described PH as a state of ego dissolution with the loss of the ability to distinguish reality from imagination [11].

Initially there was a diagnostic dilemma for our case. Part of our differential was Charles-Bonnet syndrome [12] for this case. The syndrome occurs most commonly in elderly presenting with visual impairment. The common conditions leading to this syndrome are age related macular degeneration, followed by glaucoma and cataract. But these are not accompanied by any feature of psychosis, impaired sensorium, dementia, intoxication, metabolic derangement, or focal neurological illness. Our patient came with altered sensorium. Even though she had ocular pathology relevant neurological, ophthalmological and radiological consultation directed us towards Peduncular Hallucnosis possibly due to Glioblastoma multiforme in the pons.

The clinical syndrome of "peduncular hallucinosis" consists of formed, vivid, visual hallucinations associated with sleep cycle disturbance and cranial nerve palsy. But in the absence of localizing focal neurologic deficits, it is easily confused with delirium or psychosis. When hallucination dominates the clinical presentation, a patient may be misdiagnosed as suffering from a psychiatric disorder. Visual hallucinations typically can be a cause of distress and anxiety in patients. Most of the time these patients are not diagnosed, which leads to inappropriate management and over medication. It may also cause early institutionalization. Evidence for pharmacological treatment for peduncular hallucinosis is limited. Studies showed that anticonvulsant, serotonergic agents, antipsychotics have showed some improvement [11]. But treatment with medications depends on patient's clinical condition and symptomatology. Our patient was given psychoeducation about her illness. She responded well to beside supportive psychotherapy. But unfortunately she did not follow-up in the outpatient clinic and we lost contact.

\section{References}

1. Holroyd S. Visual hallucinations in a geriatric psychiatry clinic: prevalence and associated diagnoses. J Geriatr Psychiatry Neurol. 1996;9(4):171-175.

2. Wolff HG, Curran D. Nature of delirium and allied states. Arch Neurol Psychiatr. 1935;33:1175-1215.

3. Webster R, Holroyd S. Prevalence of psychotic symptoms in delirium. Psychosomatics. 2000;41(6):519-522.

4. Dunn DW, Weisberg LA, Nadell J. Peduncular hallucinations caused by brainstem compression. Neurology. 1983;33(10):1360-1361.

5. Kumar R, Behari S, Wahi J, Banerji D, Sharma K. Peduncular hallucinosis: an unusual sequel to surgical intervention in the suprasellar region. Br J Neurosurg. 1999;13(5):500-503.

6. Geller TJ, Bellur SN. Peduncular hallucinosis: magnetic resonance imaging confirmation of mesencephalic infarction during life. Ann Neurol. 1987;21(6):602-604.

7. Feinberg WM, Rapcsak SZ. 'Peduncular hallucinosis' following paramedian thalamic infarction. Neurology. 1989;39(11):1535-1536.

8. McKee AC, Levine DN, Kowall NW, Richardson EP, Jr. Peduncular hallucinosis associated with isolated infarction of the substantia nigra pars reticulata. Ann Neurol. 1990;27(5):500-504.

9. Beckstead RM, Domesick VB, Nauta WJ. Efferent connections of the substantia nigra and ventral tegmental area in the rat. Brain Res. 1979;175(2):191-217.

10. Jacob A, Prasad S, Boggild M, Chandratre S. Charles Bonnet syndrome--elderly people and visual hallucinations. BMJ. 2004;328(7455):1552-1554.

11. Kumar R, Kaur A. Peduncular hallucinosis : an unusual sequelae of medulloblastoma surgery. Neurol India. 2000;48(2):183-185.

12. Spiegel D, Barber J, Somova M. A potential case of peduncular hallucinosis treated successfully with olanzapine. Clin Schizophr Relat Psychoses. 2011;5(1):50-53. 